\title{
Treatment of femur shaft fractures using Perkins' traction at Addis Ababa University: An Ethiopian experience
}

\author{
B. Bezabeh, M.D, Assistant Professor, and B. L. Wamisho, M.D, FCS (ECSA), Assistant Professor, Department of \\ Orthopaedic Surgery, Faculty of Medicine, Addis Ababa University, Addis Ababa, Ethiopia
}

Correspondences to: Dr. B. L. Wamisho, P.O. Box 122201, Addis Ababa, Ethiopia. E-mail: Ibiruklw@yahoo.com

\section{ABSTRACT}

Objectives: To evaluate the outcomes of Perkins' technique in the treatment of adult femur shaft fractures from October 2007 - May 2009. Faculty of Medicine, Black-Lion Hospital (BLH), Addis Ababa University, Ethiopia, The study also aimed at looking into the spectrum of femur shaft fractures referred to $B L H$.

Design: This was a prospective study.

Setting: Addis Ababa University, Black-Lion ('Tikur Anbessa') Hospital-BLH, is the country's highest tertiary level referral and teaching Hospital. The 67- bedded orthopaedic department, only one in the country, runs under-graduate and post-graduate/ residency programmes and receives referred patients from all over the country.

Methods: All the 68 consecutive femur shaft fracture adult patients admitted to the department's wards during the study period were recruited for the study. They were prospectively followed after obtaining their consents. Standard Perkins' system of traction was applied and the orthopaedic team composed of consultants, residents, physiotherapists and nurses using a Perkins' format/protocol prepared by the researchers followed patients. A physiotherapist attended each patient individually and as a group with similar patients. Knee and quadriceps exercise was done four times a day and recorded.Variables like patient's demography, cause of the fracture, characteristics of fracture, duration of traction, thigh circumference, knee range of motion, limb length, pin tract condition, complications were included in the format chart. These were recorded every week and fed into computer. Final outcomes at the end of traction were documented and analyzed. Traction is removed when there is clinical and radiological evidence of fracture union. Physiotherapists give gait training and serial check X-rays were taken at out patient fracture clinics.

Results: From the total of 68 consecutive patients admitted with femur shaft fractures and followed, 60 (88.2\%) were males and only 8 (11.8\%) were females making the fracture over eight times frequent in the male sex. About half of the patients lied in the age range 18-28 years. Road traffic accident was cause of fracture in nearly half $(49.2 \%)$ patients. The right side was more commonly fractured (40, $\mathbf{5 8 . 8} \%)$ than the left $(28,41.2 \%)$. Two thirds of the fractures $(44,64.7 \%)$ were closed. There was one bilateral fracture encountered. Half of the fractures $(34,50 \%)$ occurred in the proximal third of the femur. Transverse fracture was the commonest pattern $(29,42.6 \%)$, followed by comminuted pattern $(18,26.5 \%)$. Only three segmental fractures were admitted. Mean hospital stay was 45 days and in the majority $(33,48.5 \%)$, duration of traction was between 30- 40 days and only eight patients were on traction for more than two months. Shortly at the end of traction; circumference of thigh was reduced only in $8(11.8 \%)$ patients, knee range of motion was more than 90 degrees in $7(10.3 \%)$ patients. At a mean follow-up of eight months (range 4-20 months), only one patient ended up with non-union and there was also only one mal-union. Shortening of over $2 \mathrm{~cm}$ was noted in $11(16.2 \%)$ patients. Over all pin tract infection rate was $\mathbf{1 1 . 8} \%$, eight patients only.

Conclusion: Outcomes of conservative treatment of femur shaft fracture using Perkins' method are safe, easy, effective and very encouraging in a developing set-up like ours. There is an excellent practice of Perkins' traction at Black Lion Hospital, Addis Ababa. 


\section{INTRODUCTION}

Adult femur shaft fractures can be treated using many different ways, conservative or surgical. The choice of which method to use considers several factors including:surgeon's experience, availability of implants, cost, desired anatomical and functional outcomes and complication rates (1-4). In developing countries, lack of well-trained surgeons and other health care providers, of appropriate and affordable equipment and implants, and of reliably clean surgical environments increases the risks of surgical complications of orthopaedic procedures often to unacceptable levels. Conservative management of femur fractures with traction remains the best and very often the only option for cost-sensitive developing countries.

Traction has been used for years and the underlying central principle is the same: alignment of a long bone fracture can be achieved and maintained by continuous isotonic pulling on the soft tissue envelope along its longitudinal axis (5). In general, skeletal traction is preferred to skin traction for adult femoral shaft fractures. Different configurations, with or without suspension or frames, have been advocated. These include: straight longitudinal (Buck) traction (6), with rigid frame (Bohler-Braun's frame) (5), or some combination of mobility and suspension, such as those of Thomas-Pearson (7) or Russell (8). Among the proponents of traction, one area in particular has long been controversial: does movement at the fracture site promote or prevent bone healing? Rest and immobilization was advocated for a long time by renowned orthopaedic leaders such as Watson-Jones, John Hilton and Apley (5,9) George Perkins (1892-1979), a brilliant orthopaedic surgeon who served both world wars, started to question this orthodoxy in his famous lecture "Rest and movement" In 1953, George Perkins, making "no apology for questioning orthodoxy," introduced his technique of starting a very early active range of motion of the knee for adults in tibial traction for femoral shaft fractures (10). He believed the pushpull action of active muscular contractions on fracture fragments were beneficial to the inflammatory process, and stimulated osteogenesis (11). We know now this to be largely true and the principle is even applied in modern internal fixation techniques such as elastic or flexible nailing, and less-invasive surgical stabilization (LISS) procedures. This principle has also been applied in developing countries, where traction is very often the only possible treatment for femur fractures (12)."In 1981,
Moulton et al. reported their series of 45 consecutive patients with femoral shaft fractures treated by straight longitudinal traction, supplemented by functional bracing at around six weeks (13). All fractures healed, one with a varus malunion. The average length of stay (LOS) was eight weeks, and at six months, the average knee flexion was $127^{\circ}$. May and Neufeld reported good results with their use of a single rope suspension dynamic traction method (14). Gates modified and adapted this technique to fit the needs and materials available in developing countries (15). The Perkins' method is even simpler. It does not require a hinged cast brace or an overhead Balkan-type frame. When comfortable and safe with ambulation, the patient is discharged with instructions to keep using his crutches until he comes back for clinical and radiological followup six weeks later. The Orthopaedic department of Addis Ababa University was established over two decades back and has been running residency programme in general orthopaedics since then. It is the only department in a country of 75 million and ranks high in road traffic accident case fatalities. It has relatively better infrastructure and is better staffed compared to similar African countries. The department's overall severe deep implant infection rate was less than $1 \%$, which is better as compared to similar set-ups in East Africa. Average hospital stay as published five years back from our department was 35.4 days (16). With the current expansions and commitment, it seems it has a great potential to address Ethiopia's orthopaedic problems. Last year the department received about 150 femur fractures, of which some are lucky to get admitted for treatment. Most femur fractures are treated conservatively and interlocking nails, K-nails and plates are reserved for selected fratures. The main constraints of the department are shortage of operating time, beds and implants. Reactivation of Perkins' technique started with the rejoining the department of one of the authors who has extensive international experience in Africa and Asia. Currently the technique is alternatively used to address all closed or open femoral shaft adult extrarticular fractures, mainly traumatic.The purpose of this prospective study was to analyze the outcomes of this method in this set-up and to identify problems that could be prevented.

\section{OBJECTIVES}

This was a prospective study with objectives of:

- Evaluating the out comes of Perkins' technique for treatment of adult femur shaft fractures. 
- Looking into the spectrum of femur fractures referred to Black-Lion Hospital.

\section{PATIENTS AND METHODS}

All consecutive traumatic adult femur shaft fractures admitted to the separate traction beds in the orthopaedic wards were included. Paediatric (age>16 years), pathological and intrarticular fractures were excluded from the study.

Perkins' method: After initial clinical diagnosis and radiological analysis of the fracture pattern, simple Steinmann pin is inserted under local anaesthesia just below the tibial tubercle when the patient is admitted. Wound debridement and lavage is done under general anaesthesia if the fracture is open. Straight traction with an initial weight of 3-4 kg is applied, with the foot of the bed elevated on wooden blocks. Limb length is measured using tape maters and compared to the normal limb. If the fractured limb is longer, distraction is suspected and additional X-ray taken while the limb is in traction, and the weight is reduced. If there is shortening, more weight is added for few days. A posterior bump/ pillow may be used for posterior angulation greater than $10-15^{\circ}$. No underneath pillows in proximal third fractures. A special format/chart developed by the experienced senior author was discussed among the orthopaedic team and the patient is followed using this format. This format/protocol consists of variables like: patient's demography, cause of the fracture, characteristics of fracture, duration of traction, thigh circumference, knee range of motion, limb length, pin tract condition and any complication. Patient does knee flexion-extension exercise four times a day (8 hourly) for 30 minutes in each session. Two of these sessions are attended by a physiotherapist while the other two sessions are done by the patient alone or by the group of similar patients located in a separate "Perkins' corner" in the ward. The format is posted on a visible site on the wall next to the patient and was filled every day. The pin sites are covered and cleaned daily with antiseptics. Usually, within 3 or 4 days from admission and almost always within a week, active range of motion exercises of the knee are started: the distal half of the bed is broken to $90^{\circ}$ or removed, and the patient is encouraged to sit while a therapist cups the affected heel to support the leg. Then, with the weight still pulling, gentle active flexion and extension is started. Pain treatment may be necessary for the first few days, but pain levels become tolerable with surprising rapidity.The flexion/extension exercise regimen is performed for half an hour, 2-4 times a day, preferably by physiotherapist. The patient is encouraged to exercise as tolerated. Group of similar patients could train and encourage each other in the ward. At this point, active flexion of the knee usually reaches $90^{\circ}$.The important steps in the Perkins' method are shown on Figures 3-7.

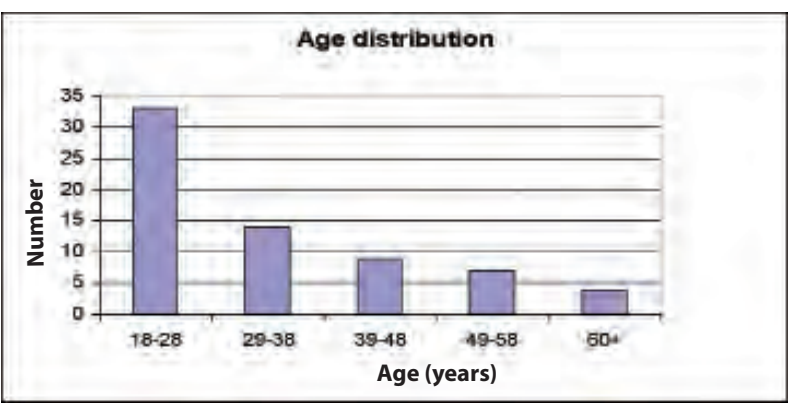

Figure 1: Initial X-ray

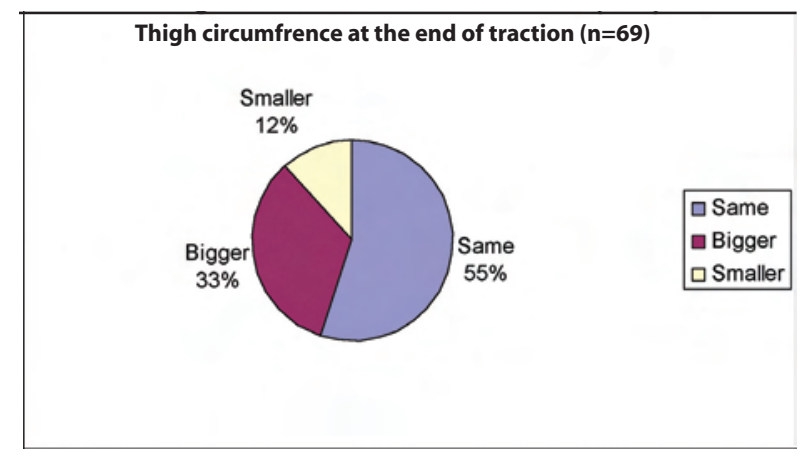

Figure 2: Final X-ray

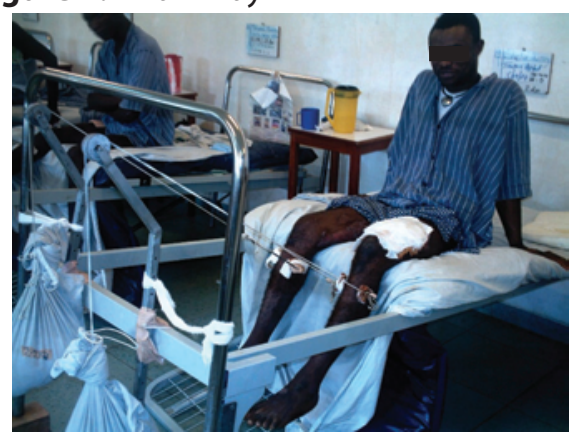

Figure 3: Perkin's system with knee flexed

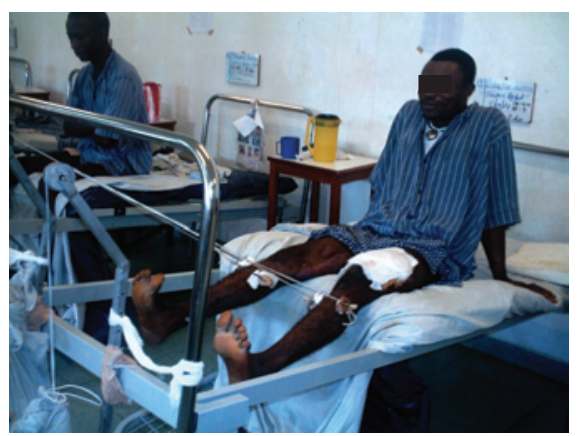

Figure 4: Perkin's system with knee extended 


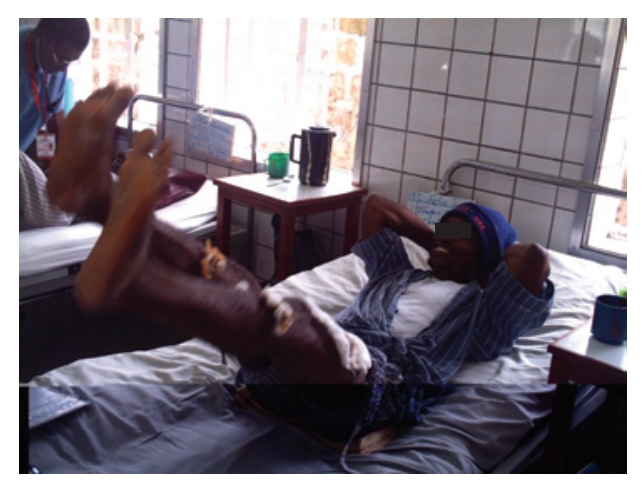

Figure 5: Clinical union, straight leg test

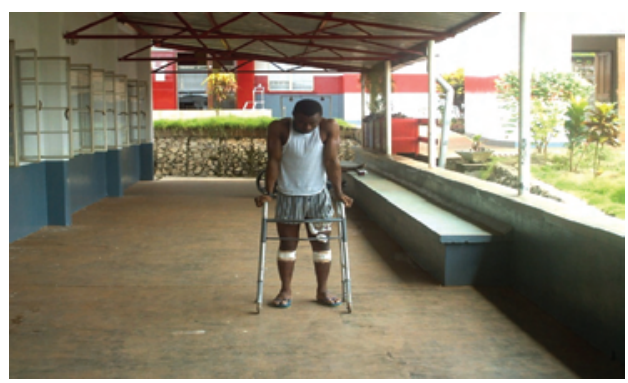

Figure 6: Bearing weight

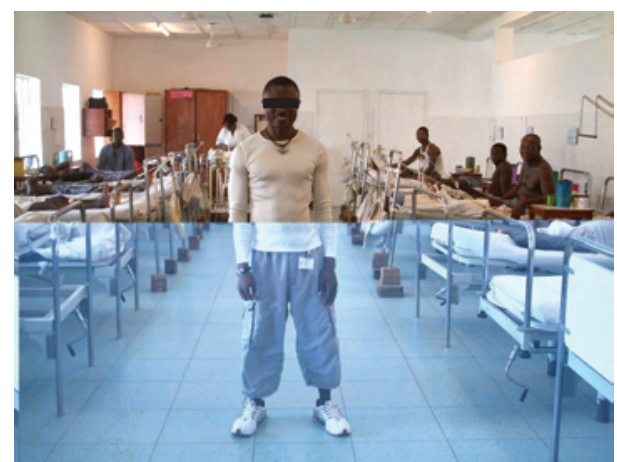

Figure 7: Patient walking

The hip musculature can be further exercised with the patient standing upright on his opposite limb. At six weeks, clinical healing is assessed by palpation of the callus, feeling for motion at the fracture site, and determining if the patient is capable of straight leg raising (except proximal third fractures). X-rays are taken. If clinical and/or radiological healing is not satisfactory, traction is reapplied, and the same evaluation is repeated every two weeks. If healing appears satisfactory, traction is discontinued, and mobilization is started using walker frames or crutches. A cast or preferably a hinged cast brace may be used, if available, for added protection. Usually, in the otherwise healthy, young, and compliant patient, protected ambulation with crutches and toe-touch weight bearing is started the next day after removing the traction. When comfortable and safe with ambulation, the patient is discharged with instructions to keep using his crutches until he comes back for clinical and radiological follow-up six weeks later to the fracture clinics. Consolidation was assessed clinically by looking for motion at the fracture site, the ability to do straight leg rising (except in proximal third fractures) without deformity or pain, and later on, pain with weight bearing. All patients in the Perkins group had X-rays taken before discharge and at return follow-ups. A non-union was diagnosed if there was still motion at the fracture at last follow-up. Healing with more than $10^{\circ}$ of varus or valgus in the frontal plane, or $15^{\circ}$ of anterior/posterior angulation in the sagittal plane was considered a malunion. A pin tract was considered infected if there was purulent discharge, loosening or ring sequestrum on X-rays, but not if there were only local inflammatory signs. Statistical analysis was done using Excel and SPSS version 11.

\section{RESULTS}

After exclusions by criteria, a total of 68 patients were enrolled and from the consecutive adult patients admitted with femur shaft fractures and followed, 60 (88.2\%) were males and only $8(11.8 \%)$ were females making the fracture over eight times frequent in the male sex.

Table 1

Distribution of pattern of adult femur shaft fractures treated using Perkins' method at Black-Lion Hospital

\begin{tabular}{lcc}
\hline Pattern of fracture & No. & (\%) \\
\hline Transverse & 27 & 39.7 \\
Comminuted & 18 & 26.5 \\
Oblique & 12 & 17.6 \\
Spiral & 7 & 10.3 \\
Segmental & 3 & 4.4 \\
Bilateral & 1 & 1.5 \\
\hline Total & 68 & 100 \\
\hline
\end{tabular}


Table 2

Distribution of causes of all adult femur shaft fractures presented to $B L H$, radiology department

\begin{tabular}{lcc}
\hline Cause & Frequency & $(\%)$ \\
\hline RTA & 202 & 47.9 \\
Fall & 126 & 29.9 \\
Machine & 28 & 6.6 \\
Blow/assault & 21 & 5.0 \\
Crush & 10 & 2.4 \\
Bullet & 19 & 4.5 \\
Hit by stick & 13 & 3.1 \\
Pathological & 2 & 0.5 \\
Stab & 1 & 0.2 \\
\hline Total & 422 & 100 \\
\hline
\end{tabular}

Table 3

Duration of hospital stay of adult femur shaft fractures treated using Perkins' method at BLH

\begin{tabular}{lcc}
\hline Days & No. & $(\%)$ \\
\hline $30-40$ & 33 & 48.5 \\
$41-50$ & 10 & 14.7 \\
$51-60$ & 17 & 25.0 \\
$60+$ & 8 & 11.8 \\
\hline Total & 68 & 100 \\
\hline
\end{tabular}

Figure 1 shows that about half of the patients lied in the age range of 18-28 years. Road traffic accident was cause of fracture in nearly half (49.2\%) of the patients. Table 2 displays distribution of the causes and pattern of all adult femur fractures presented to radiology department in the same faculty in a half-year period just before the commencement of our study (March-August, 2007). The right side was more commonly fractured $(40,58.8 \%)$ than the left $(28,41.2 \%)$. Two thirds of the fractures $(44,64.7 \%)$ were closed. There was one bilateral fracture and the initial and final $\mathrm{X}$-rays of this patient is shown on Figures 8-9. Half of the fractures (34, $50 \%$ ) occurred in the proximal third of the femur. Transverse fracture was the commonest pattern $(29,42.6 \%)$; followed by comminuted pattern (18, $26.5 \%)$.

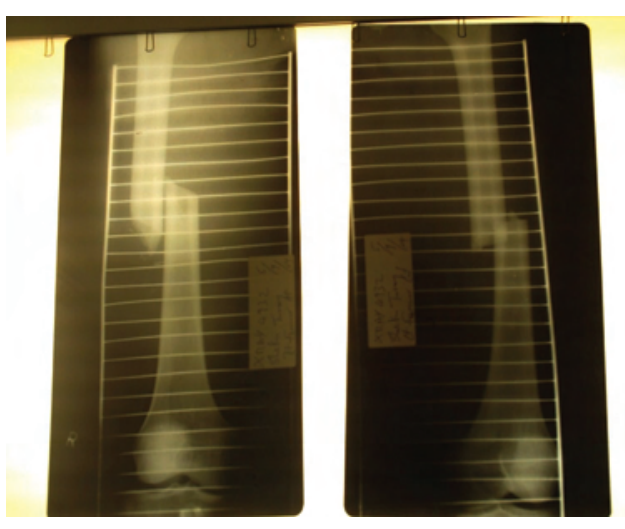

Figure 8: Age distribution of adult femur shaft fractures treated using Perkins' method at BLH

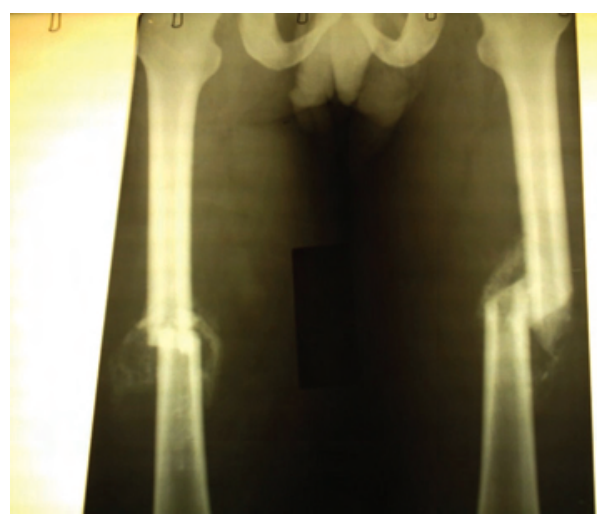

Figure 9: Comparison of thigh circumference at the end of treatment of adult femur shaft fractures treated using Perkins' method at BLH

The remaining fracture patterns are shown on Table 1. Only three segmental fractures were admitted. Mean hospital stay was 45 days and in the majority $(33,48.5 \%)$, duration of traction was between 30- 40 days and only eight patients were on traction for more than two months. Duration of hospital stay is shown by Table 3. Shortly at the end of traction; circumference of thigh was reduced only in $8(11.8 \%)$ patients, the distribution is shown by Figure 2 . knee range of motion was 40-60 degrees in ten patients and between $60-90$ degrees in 50 patients. It was more than 90 degrees in 7 (10.3\%) patients. At mean follow-up of eight months (range 4- 21 months). Only one patient ended up with non-union and there was also only one mal-union. Shortening of over $2 \mathrm{~cm}$ was noted in 11(16.2\%) patients. Over-all pin tract infection rate was $11.8 \%$, eight patients only. 


\section{DISCUSSION}

Forty years back, developed countries shifted towards surgical treatment of long bone fractures and today, generally surgical fixation gives better results than conservative treatment, in terms of anatomical and functional outcomes, complication rates, and even costs, particularly in the polytrauma patients (1-4). This is true of high income, developed countries, where almost all the studies have been carried out, but not necessarily the case for poorer nations. The lack of well-trained surgeons and other health care providers, of appropriate and affordable equipment and implants, and of reliably clean surgical environments increases the risks of surgical complications of orthopaedic procedures often to unacceptable levels. Conservative management of femur fractures with traction remains the best and very often the only option for resourcepoor countries. Conservative management of adult femoral shaft fractures is now used almost exclusively in environments where resources are significantly limited. This will remain the case until human, technical, and infrastructural capacity to carry out surgical fixation in a safe, reliable, and sustainable fashion is available. Union rates are consistently reported as being above $90 \%$ (1619). Prolonged hospital stay and rehabilitation, with their associated direct and indirect costs, are usually not as much an issue as in richer countries. In the transitional environments of developing economies, the risks and benefits of conservative treatment still need to be weighed against those of surgical fixation by overly enthusiastic, but inadequately trained hands working in less than favorable conditions. Complications such as malunion, excessive shortening, and re-fractures are more or less in line with data reported from many other Perkins'series. In our report, the pin tract infection rate of $11.8 \%$ for all Perkins' procedures is compared to other studies and was found to be: the same to a $-15 \%$ report (18), higher than an $8.6 \%$ report (19), and much lower than a $36 \%(6)$ and $41.7 \%$ (20) Once a patient is able to flex the knee about 90 degrees, permanent functional disability will be insignificant. (21). A study conducted in our department in 2003 revealed that the mean duration of stay in hospital between admission and elective operation was 11.7 days. The post-operative stay was 20.6 days and the average length of hospital stay of all patients admitted was 35.4 days. The bed occupancy rate was $97.4 \%$ with an average time between the discharge of one patient and the admission of another (turnover interval) of 24 hours. According to a study recently published from our hospital, the radiology department received 68 adult femoral fractures $(68 / 422,15.1 \%$ of all fractures) just in half a year, March-August 2007 and road traffic accident constitutes the largest proportion, 202(47.9\%) among traumatic causes, followed by fall down accident $126(29.9 \%)$ and blow assaults $28(6.6 \%)$ (Table-3) (22). The distribution of the site of fractures in this study at the radiology department ( $n=68$, proximal shaft $44.1 \%$, middle shaft 41.1, distal shaft 11.7 and intra-articular $2.9 \%$ is comparable with our study) even though it is done few months earlier than our study ( $n=68$, proximal shaft $44.1 \%$, middle shaft $41.1 \%$, distal shaft $11.7 \%$ and intra-articular 2.9\%). Since other complications are in line with other published reports, and healing in a good position can be achieved nearly over $95 \%$ of the time, with anecdotally better knee motion than other methods, the Perkins', method seems a safe and valuable treatment option for this particular environment.

\section{CONCLUSION}

The outcomes of Perkins' method of treating femur shaft fractures in an adult at the orthopaedic department in Addis Ababa University, Ethiopia are encouraging. Perkins' technique remains safe, effective, cheaper and best alternative way of addressing femur shaft adult fractures in cost-sensitive developing nations where there is also less number of experienced operating staff implants and theatre facilities.

\section{RECOMMENDATIONS}

Based on the findings of this study on treatment of traumatic femur shaft fracture in an adult, we recommend the following:

- When personally well followed with commitment, Perkins' technique is the best alternative way of addressing uncomplicated femur shaft fractures in resource-poor countries, in well motivated patients.

- Surgical treatment is reserved for some intraarticular fractures, established non-unions or unacceptable malunions.

- Exposing Orthopaedic and surgical trainees in developing countries to different methods of fracture treatment is advantageous. 
- Group exercise by similar patients in a separate ward/bay may motivate and encourage patients to adhere to Perkins' treatment protocol.

- Smaller weight on traction ( $3 \mathrm{~kg}$ ) for African and Asian patients may decrease the rate of nonunion from distraction, and may need no or less number of check X-rays while on traction.

- Simple format/protocol to document and monitor Perkins' technique makes follow-up of outcomes easy.

- In terms of complications, removing traction too early is worse than leaving it too long.

\section{ACKNOWLEDGEMENTS}

We thank the staff in the department of orthopaedics and appreciate our patients' co-operation in the study.

\section{REFERENCES}

1. Bucholz, R.W. and Jones, A. Fractures of the shaft of the femur. J. Bone J. Surg. Am. 1991; 73-A: 1561-1566.

2. Fakhry, S.M., Rutledge, R., Dahners, L.E. and Kessler, D. Incidence, management, and outcome of femoral shaft fracture: a statewide population-based analysis of 2805 adult patients in a rural state. J. Trauma. 1994; 37: 255261.

3. Gillquist, J., liljedahl, S.O. and Rieger, A. Fracture of the femur diaphysis. Traction or nailing? Acta Chir Scand. 1971; 137: 515-520.

4. Pape, H.C., Hildebrand, F., Pertschy, S., Zelle, B., et al. Changes in the management of femoral shaft fractures in polytrauma patients: from early care to damage control orthopedic surgery. J. Orthop. Trauma. 2004; 18 (8 Suppl): S13-S23.

5. Peltier, L.F. A brief history of traction. J. Bone J. Surg. Am. 1968; 50-A (8): 1603-1616.

6. Buxton, R.A. The use of Perkins' traction in the treatment of femoral shaft fractures. J. Bone J. Surg. Br. 1981; 63-B: 362-366.
7. Bryson, A.F. Treatment of fractures of the femur. Trop. Doct. 1971; 1: 68-71.

8. Anderson, R.L. Conservative treatment of fractures of the femur. J. Bone J. Surg. Am. 1967; 49-A: 1371-1375.

9. Apley, A.G. A system of orthopedics and fractures, 1 st edn. Buttersmith, London. 1959;

10. Perkins, G. Rest and movement. J. Bone J. Surg. Br. 1953; 35-B:521-540.

11. Perkins, G. Fractures and dislocations. University of London, Athlone Press, London. 1958.

12. Kevau, I. and Watters, D.A. Conservative management of femoral shaft fractures. PNG Med. J. 1996; 39: 143-151.

13. Moulton, A., Agunwa, W.C. and Hopkins, J.S. Closed femoral fractures: why not simple traction? Injury. 1981; 13: 244-248.

14. Mays, J. and Neufeld, A.J. Skeletal traction methods. Clin. Orthop. Relat. Res. 1974; 102:144-151

15. Gates, D.J., Alms, M. and Cruz, M.M. Hinged cast and roller traction for fractured femur. A system of treatment for the third world. J. Bone J. Surg. 1985; 67-B: 750-756.

16. Ahmed, A. and Chaka, E, T. Orthopedic and major limb trauma at the Tikur Anbessa University Hospital. East Afr. Med. J. 2005; 10: 43 - 50.

17. Mandrella, B. The conservative treatment of femur fractures by Perkins' traction. Unfallchirug. 2002. 105: 923-931.

18. Procter, D.S.C. The functional treatment of fractures of the femur. S. Afr. Med. J. 1962. 36: 598-602.

19. Usdin, J. The treatment of fractures of the femur by Perkins' traction. S. Afr. Med. J. 1969; 43: 480-482.

20. Richard, G. and Daniel, L. Perkins traction for adult femoral shaft fractures: a report on 53 patients in Sierra Leone. Int. Orthop. 2007; 31(5): 697-702.

21. Lambisso, W. Biruk, Permanent Civilian musculoskeletal disability following injury-17 year trends. ECASJ. 2006; 11: 41-48.

22. Admasie, Y., Tekle, Y, B.L.Wamisho - Radiological and clinical details of major adult limb fractures in a Teaching Hospital, AAU, Ethiopia. ECAJS. 2009; 14: 88-97. 